\title{
The qualitative criterion of transient angle stability
}

Lyu, R.; Xue, Y.; Xue, F.; Wu, Qiuwei; Yang, Guangya; Zhou, H.; Ju, P.

Published in:

Proceedings of The 10th International Conference on Advances in Power System Control, Operation \& Management

Publication date:

2015

Document Version

Peer reviewed version

Link back to DTU Orbit

Citation (APA):

Lyu, R., Xue, Y., Xue, F., Wu, Q., Yang, G., Zhou, H., \& Ju, P. (2015). The qualitative criterion of transient angle stability. In Proceedings of The 10th International Conference on Advances in Power System Control, Operation \& Management IEEE.

\section{General rights}

Copyright and moral rights for the publications made accessible in the public portal are retained by the authors and/or other copyright owners and it is a condition of accessing publications that users recognise and abide by the legal requirements associated with these rights.

- Users may download and print one copy of any publication from the public portal for the purpose of private study or research.

- You may not further distribute the material or use it for any profit-making activity or commercial gain

- You may freely distribute the URL identifying the publication in the public portal

If you believe that this document breaches copyright please contact us providing details, and we will remove access to the work immediately and investigate your claim 


\title{
THE QUALITATIVE CRITERION OF TRANSIENT ANGLE STABILITY
}

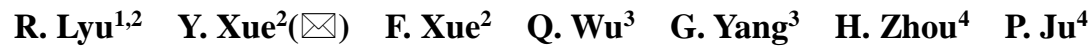 \\ xueyusheng@sgepri.sgcc.com.cn \\ 1 Nanjing University of Science and Technology, Nanjing 210094, China \\ 2 State Grid Electric Power Research Institute, Nanjing 210003, China \\ 3 Technical University of Denmark, Lyngby 2800, Denmark \\ 4 Hohai University, Nanjing 210098, China
}

\begin{abstract}
In almost all the literatures, the qualitative assessment of transient angle stability extracts the angle information of generators based on the swing curve. As the angle (or angle difference) of concern and the threshold value rely strongly on the engineering experience, the validity and robust of these criterions are weak. Based on the stability mechanism from the extended equal area criterion (EEAC) theory and combining with abundant simulations of real system, this paper analyzes the criterions in most literatures and finds that the results could be too conservative or too optimistic. It is concluded that misjudgment would be taken if an angle (or angle difference) of concern departing from the concept of the controlling mode or a constant threshold value is used in the criterion.
\end{abstract}

KEYWORDS: Transient angle stability criterion, angle (or angle difference) of concern, angle threshold value, EEAC

\section{INTRODUCTION}

The interconnected power grid develops greatly on cover area, voltage level, transmission capacity and the like. It brings a lot of economic benefit, however it also increases the accident probability of power system. This serious situation requires higher security and better stability of power system, therefore it becomes an emphasis and difficulty in the research of power system security.

Transient angle stability criterion is the basis to qualitatively analyze the stability of dynamic simulation curves. It is one of the key points for the mechanism research of power system security, and an important foundation for the planning and operation optimization algorithm. In many fields such as available transfer capability (ATC) [1] and optimal power flow (OPF) [2], the qualitative criterions are normally shown in the form of inequality to represent transient angle stability constraint.

In almost all the literatures, the angle (or angle difference) of concern in the qualitative criterions are defined as the maximal angle difference between any two generators [3] or the maximal angle difference between any generator and the center of inertia (COI) [1,2,4-15]. However, in some literatures, the multimachine system is transformed into several equivalent or specific generators, and then the above two methods are used to set the angle (or angle difference) of concern [16,17]. Since all these methods are not based on the transient stability mechanism to obtain the angle (or angle difference) of concern, they cannot reflect the transient stability behaviors of the system. Moreover, in almost all the literatures, the angle threshold value is experimentally set as a large enough constant which is unrelated to the topological structure, parameter, operating condition or fault scenario of power system. Therefore, some transient unstable cases may be misjudged as stable due to the short observation period, and some transient stable cases may be misjudged as unstable due to a threshold value which is not robust.

EEAC theory utilizes the complementary-cluster center-of-inertia relative-motion (CCCOI-RM) transformation, which is a full rank linear transformation, to map the complete disturbed trajectories into a series of equivalent (variable parameter) trajectories of one-machine-infinity-bus (OMIB) systems, so that the complete stability information of the original multi-machine system can be reserved [18]. On the image trajectory, EEAC points out that it is judged as unstable if there exists the dynamic saddle point (DSP) on the power-angle $(P-\delta)$ curve of the time-varying image system, or stable if there exists the far end point (FEP). DSP and FEP will be coincident on the critical trajectory. Finally, we can obtain the necessary and sufficient condition and critical parameters of the image system by sensitivity technology [19].

Among all the image systems, the one with the most rigorous critical parameter is defined as the critical mode. Thus identifying the critical mode is an iterative process. The concerned angle (or angle difference) and the angle threshold value have to be automatically searched according to the specific case. It has been theoretically proved that EEAC which strictly reflects the necessary and sufficient condition of stability has a clear physical significance [20]. 
This paper compares the qualitative criterions of transient angle stability with that of EEAC and other researches. The simulations of real systems show that even in the same system and same operation condition, the critical angle threshold value of different case could be different. It means that the stability, even the qualitative result, cannot be guaranteed if the angle threshold value is set as a constant. In conclusion, it will cause a serious consequence if a constant threshold value or an angle (or angle difference) of concern departing from the concept of the controlling mode is used in the criterion.

\section{REVIEW OF THE QUALITATIVE CRITERION OF TRANSIENT ANGLE STABILITY}

\subsection{Qualitative Criterions in Most Literatures}

The transient stability constraints have been explained in most literatures by the qualitative criterions in the following two forms:

1. In the transient process, with the criterion that the angle difference between any two generators does not exceed a certain limit, the transient stability constraint is:

$$
\delta_{\min } \leq \delta_{i}-\delta_{j} \leq \delta_{\max }, \quad i, j=1,2, \cdots, n_{g}
$$

where $\delta_{i}$ and $\delta_{j}$ are the angle of the generator $i$ and $j$ repetitively, $\delta_{\min }$ and $\delta_{\max }$ are the threshold value of $\delta_{i}$ and $\delta_{j}$ repetitively and $n_{g}$ is the amount of the total generators.

2. In the transient process, with the criterion that the angle difference between any generator and the COI does not exceed a certain limit, the transient stability constraint is:

$$
\delta_{\min } \leq \delta_{i}-\delta_{\mathrm{COI}} \leq \delta_{\max }, \quad i, j=1,2, \cdots, n_{g}
$$

where $\delta_{\mathrm{COI}}$ is the angle of the COI. Donate $M_{i}$ as the moment of inertia of the generator $i$,

$$
\delta_{\mathrm{COI}}=\frac{\sum_{i=1}^{n_{g}} M_{i} \delta_{i}}{\sum_{i=1}^{n_{g}} M_{i}} .
$$

However, the two forms above rely strongly on the swing curves of partial generators [3], namely the complete information of all the generators is not fully considered, and it is divorced from the concept of the controlling mode. Therefore the two forms of criterions above cannot be necessary or sufficient to reflect the stability of the original system.

In addition, some other literatures propose a "pregrouping offline, online dynamic correction" method $[16,17]$ : Firstly, the whole generators are divided into several sub-clusters during the observation period. In this process, it has to be ensured that in each subcluster, the maximal of angle difference between any two generators does not exceed a preset limit. Secondly, the generator with the most stable swing curve is selected as the center generator of its subcluster, namely each sub-cluster is represented by one generator. Thirdly, the two methods mentioned hereinbefore are applied to the center generators to sentence the stability. If the angle (or angle difference) of concern exceeds the limit, the system is sentenced to be unstable. Meanwhile, in order to prevent an unexpected situation where a generator separates from its sub-cluster, all generators within a cluster will be compared with the center generator. And if the deviation exceeds the preset limit, this generator will be processed as a new sub-cluster to participate the comparison in the next moment.

However, this method does not fully consider the complete information of the system and does not follow the concept of the controlling mode. Moreover its way of selecting the center generator of each subcluster has already lost some information of the system. Therefore, it cannot reflect the necessary or sufficient conditions.

On the other hand, the angle threshold value in the literatures is mostly set as a constant, which is based on the engineering experience. For example, for Criterion 1 mentioned above, the angle threshold value is mostly set as 100 degrees and in some other literatures set as 120 or 150 degrees. For Criterion 2, the angle threshold value is mostly set as 360 degrees. Besides, the observation period is always set as 1 second or 2 seconds, which is also based on the engineering experience and system performance.

Theoretically, only if the observation period and the angle threshold value are both infinite, we can correctly judge the stability of the swing curve by observing whether it is divergent. However, the observation period is set as $1 \mathrm{~s}$ or $2 \mathrm{~s}$, the system is likely to be sentenced as stable in the case where the swing curve has not even been divergent yet during the observation period. Also, a stable case where the angle difference is too great is likely to be sentenced as unstable. In addition, short observation period could cover up some special phenomena such as resynchronization.

\subsection{The Qualitative Criterion and Quantitative Indicator of EEAC Method}


EEAC theory disassembles the transient assessment of power system into two sub-tasks: obtaining the disturbed trajectory in $R^{n}$ and analyzing the image trajectory in $R^{1}$. The former sub-task is used to ensure the model applicability and the accuracy, and the latter one is used to quantitative analyze the system and search for the stability limit. Meanwhile, the CCCOI-RM transforms is used to ensure the feasibility of the process above.

After obtaining the actual disturbed trajectory of the system through numerical integration, substituting $P_{\mathrm{m} i}(t)$ and $P_{\mathrm{e} i}(t)$ into the equation of the original multi-machine system, where $P_{\mathrm{m} i}(t)$ is the mechanical power of generator $i$ and $P_{\mathrm{e} i}(t)$ is the electromagnetic power of generator $i$, the right term of the equation becomes a known function of time without any other variables. For a given division manner of two complementation-clusters (the critical cluster is denoted as $S$, and the remaining cluster is denoted as $A$ ), it divides the motion equations of $n$ generators into two subsets. Summing up both sides of all the equations of each subset, one obtains two independent trajectories belonging to $R^{2}$. With reference to one of the two trajectories (for example cluster A), we get the numerical image of equivalent OMIB system under this division manner.

Define the point, which satisfies that

$$
\begin{gathered}
P_{\mathrm{ub}}=P_{\mathrm{m}}-P_{\mathrm{e}}=0, \\
\mathrm{~d} P_{\mathrm{ub}} / \mathrm{d} \delta>0
\end{gathered}
$$

where $P_{\mathrm{ub}}$ is the unbalanced power, $P_{\mathrm{m}}$ is the mechanical power and $P_{\mathrm{e}}$ is the electromagnetic power, as DSP of the image system.

Define the point, which satisfies that

$$
\omega=0
$$

where $\omega$ is the angular velocity, as FEP of the image system.

Analyzing the numerical image of equivalent OMIB system on the expansion phase plane, one obtains the stability margin of each swing. The equation of stability margin varies from the character of each swing, namely if the trajectory reaches FEP, the stability margin is

$$
\eta=\frac{A_{\text {dec.pot }}}{A_{\text {inc }}+A_{\text {dec.pot }}} \times 100 \%
$$

where $A_{\text {dec.pot }}$ is the potential kinetic energy decrease area and $A_{\text {inc }}$ is the kinetic energy increase area. If the trajectory exceeds DSP, the stability margin is

$$
\eta=\frac{A_{\mathrm{dec}}-A_{\mathrm{inc}}}{A_{\mathrm{inc}}} \times 100 \%
$$

where $A_{\mathrm{dec}}$ is the kinetic energy decrease area.

The minimal stability margin of all the swings is defined as the stability margin of the trajectory under the division manner. Furthermore, the minimal stability margin of all the division manners is defined as the stability margin of the original high-dimension system [19].

EEAC theory gives the necessary and sufficient conditions of the stability of multi-machine system. It provides a quantitative result and clearly reflects the mechanism of transient stability which finds and explains many complex phenomena such as the negative control effects and the isolated stability domain (ISD).

\subsection{Comparison}

The qualitative criterion of transient angle stability in most literatures is based on the swing curves, whereas EEAC theory is based on the power-angle curves. The swing curves cannot reflect the physical mechanism of the stability of multi-machine system. On the contrary, since the concept of energy is involved through the angle and the power, the power-angle curves has the ability to reflect the mechanism. Therefore, EEAC not only points out a strict qualitative criterion, but also has the ability to analyze the stability quantitatively.

\section{SIMULATIONS AND ANALYSIS}

The simulation systems are based on the Shandong Power Grid and the China Southern Power Grid. Through simulations on the various systems under different fault scenarios (fault location or clearing time), we analyze the maximal angle difference (as an example, the maximal angle difference between any two generators) of each case under the critical stable circumstance. Finally, a constant is set as the threshold value to qualitatively analyze the stability, and the result is compared with the integrating EEAC (IEEAC).

\subsection{Real Cases of Shandong Power Grid}

This system is based on the data from the Shandong Power Grid in 2004. We simulate all the 52 cases under three-phase permanent short-circuit fault on $500 \mathrm{kV}$ lines and record the maximal angle difference between any two generators under the critical stable 
circumstance.

Making these maximal angle differences into a histogram, the interval of which is $\mathbf{5}$ degrees and the vertical axis of which is the amount of cases in the relevant interval, we obtain the Fig. 1 below.

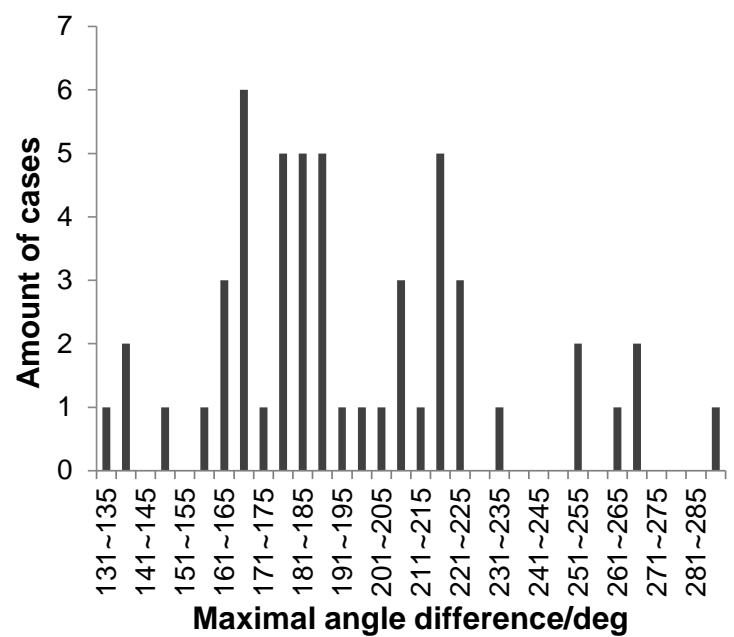

Fig. 1: The distribution of maximal angle differences of Shandong Power Grid

It comes from Fig. 1 that the 52 angle differences range from 135 degrees to 288 degrees and they distribute dispersedly without a trend of concentration.

\subsection{Real cases of China Southern Power Grid}

This system is based on the data from the China Southern Power Grid in 2011. We simulate all the 429 cases under three-phase permanent short-circuit fault on $500 \mathrm{kV}$ lines and record the maximal angle difference between any two generators in the critical stable circumstance.

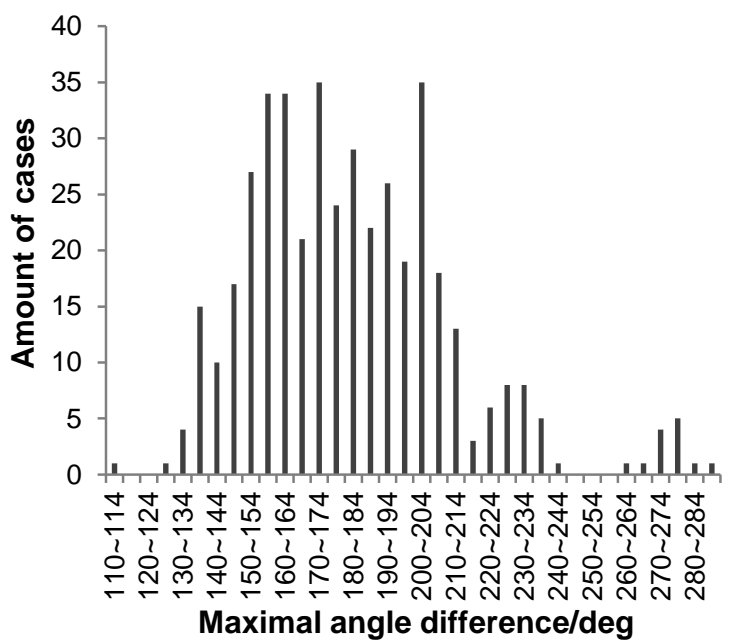

Fig. 2: The distribution of maximal angle differences of China Southern Power Grid

Making these maximal angle differences into a histogram, the interval of which is 5 degrees and the vertical axis of which is the amount of cases in the relevant interval, we obtain the Fig. 2 below.

Fig. 2 shows that the 429 angle differences range from 114 degrees to 288 degrees and they do not present a trend of concentration.

\subsection{Analysis and Inference}

Although the simulation systems are based on the real systems under the common conditions and the common fault scenarios, we can find that the maximal angle differences range widely. Therefore, if the angle threshold value is set as a constant, then it is inevitable that quite a few cases will be misjudged. For example, for the 52 simulation results of the Shandong Power Grid, the minimum value of the maximal angle differences is 135 degrees (case 14, Fig. 3) and the maximum value is 288 degrees (case 25, Fig.4). If the angle threshold value is set as a constant less than 135 degrees, then all the cases will be misjudged as unstable; If the threshold value is set as a constant belongs to $[135,288]$, then part of the cases will be misjudged as unstable. Obviously, the analyses do not conform to the fact.

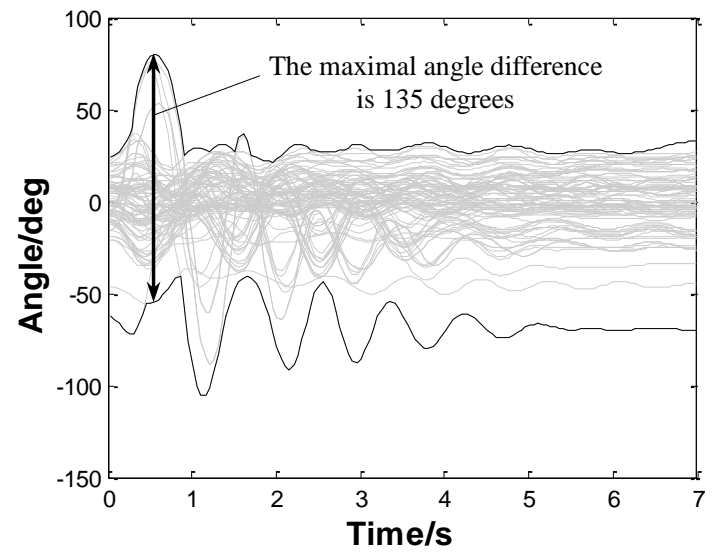

Fig. 3: The swing curve of case 14 in Shandong Power Grid

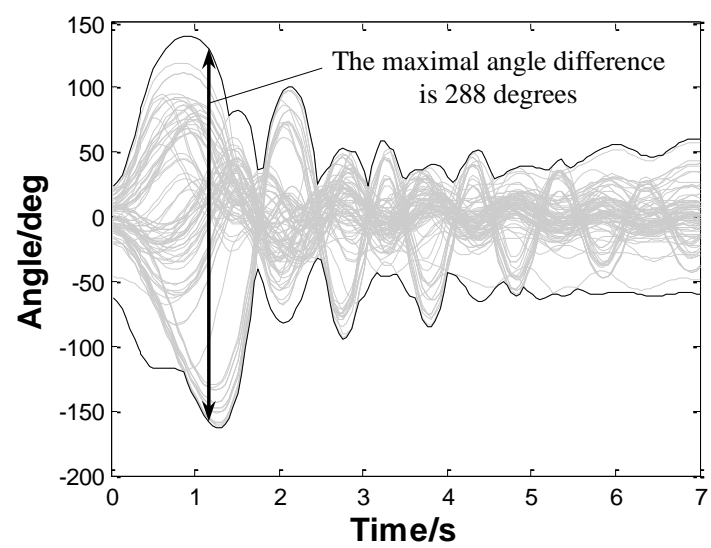

Fig. 4: The swing curve of case 25 in Shandong Power Grid 
The simulations above involve critical stable circumstances under the critical clearing time. It can be inferred in actual circumstance that the maximum angle difference may be very large in stable cases and small in unstable cases. It reflects the angle threshold value cannot be a constant, but a variable which varies with the change of many factors such as operation conditions, fault scenarios, simulation parameters and etc.

In addition, the criterions in the literatures do not have the conditions to terminate the integration in advance. The only way to decrease the possibility of misjudgments is that setting a large enough angle threshold value and a long enough observation period. The fixed observation period set by engineering experience cannot guarantee the necessary or sufficient condition and cannot decrease computational burden. However, the qualitative criterion in EEAC theory uses the disturbed trajectory reaching the DSP as a sufficient and necessary condition. Once the trajectory reaches the DSP, the integration can be terminated. In conclusion the EEAC avoids a mess of useless computation and ensues the necessary and sufficient of the stability analysis.

\section{CONCLUSIONS}

Transient stability qualitative criterion has a wide range of application. This paper analyzes the ways of setting angle (or angle difference) of concern and angle threshold value based on the mechanism of transient stability, and it is concluded that these methods have some limitations. Furthermore, through abundant simulations based on the real systems, it is verified that the angle threshold value cannot be a constant, otherwise the result may be too conservative or too aggressive. These problems should be paid attention to by the researchers.

\section{ACKNOWLEDGEMENT}

This work is jointly supported by State 973 Project (No. 2013CB228204) and SGCC Projects.

\section{REFERENCES}

[1] LIU M. et al: 'Calculation of available transfer capability with transient stability constraints', Proceedings of the CSEE, Vol.23, No.9, 2003, pp.2833.

[2] YUAN Y. et al: 'Interior-point method based optimal power flow with transient stability constraints', Automation of Electric Power System, Vol.26, No.13, 2002, pp.14-19.
[3] WU Y., LI L.: 'Transient stability constrained optimal power flow based on sensitivity of transient energy margin', Power System Technology, Vol.29, No.15, 2005, pp.28-33.

[4] WANG L. et al: 'Calculation of available transfer capability taking into account transient stability constraints based on interior-point solution in electricity market', Proceedings of the CSU-EPSA, Vol.16, No.1, 2004, pp.28-33.

[5] WEI H. et al: 'Optimal power flow with multicontingencies transient stability constraints', Proceedings of the CSEE, Vol.24, No.10, 2004, pp.91-96.

[6] YUAN Y. et al: 'Available transfer capability determination considering transient stability constraints', Automation of electric power systems, Vol.28, No.10, 2004, pp.13-17.

[7] LI L. et al: 'Calculation on optimal power flow considering transient stability constraints on tie-lines', Power System Technology, Vol.29, No.11, 2005, pp.25-29.

[8] WANG N., WEI H.: 'Calculation of Available Transfer Capability with Transient Stability Constraint', Electric Power Science and Engineering, No.1, 2005, pp.1-4.

[9] LI B. et al: 'A new method of optimal power flow calculation considering tie lines transient stability constraints', China Rural Water and Hydropower, No.5, 2005, pp.106-108.

[10] LIU Y., LI L.: 'A method calculating maximum transmission capability of tie-line considering transient stability constraints', Power System Technology, Vol.30, No.11, 2006, pp.56-61.

[11] XIAX., WEI H.: 'Transient stability constrained optimal power flow model based on three-step implicit Adams method', Automation of Electric Power System, Vol.36, No.11, 2012, pp.55-61.

[12] XIAX., WEI H.: 'A reduced-space interior point method for transient stability constrained optimal power flow in polar coordinate', Power System Protection and Control, Vol.40, No.4, 2012, pp.14-19.

[13] XIA X. et al: 'An improved reduced-dimension interior point method for transient stability constrained optimal power flow', Transactions of China Electrotechnical Society, Vol.27, No.9, 2012, pp.87-92.

[14] WEI Z. et al: 'Interior-point optimal power flow of AC-DC system with VSC-HVDC', Proceedings of 
the CSEE, Vol.32, No.19, 2012, pp.89-95.

[15] YANG Y., WEI H.: 'Recursive reduced-order and decoupling algorithm for solving transient stability constraints optimal power flow', International Conference on Power System Technology, Chengdu, China, Oct. 22-24, 2014, pp.170-175.

[16] XUE F., YIN J.: 'Forecasting for transient stability of power system based on GPS \& grouping theory', North China Electric Power, Vol.23, No.5, 2001, pp.11-12.

[17] YIN J. et al: 'Application of grouping theory in forecast and control of transient stability of power systems', Information on Electric Power, No.1, 2002, pp.1-4.

[18] XUE Y.: 'A critical comparison of various methods for transient stability assessment, part one: disturbed measure functions', Automation of Electric Power Systems, Vol.25, No.11, 2001, pp.6-11.

[19] XUE Y.: 'Quantitative study of general motion stability and an example on power system stability', Jiangsu Science and Technology Press, Nanjing, China, 1999.

[20] ZOU Y. et al: 'Synchronous stability of nonautonomous dynamic systems', Acta Mathematicae Applicatae Sinica, Vol.24, No.1, 2001, pp.155-157. 